\title{
EXISTENCE OF A NONTRIVIAL SOLUTION TO A STRONGLY INDEFINITE SEMILINEAR EQUATION
}

\author{
B. BUFFONI, L. JEANJEAN, AND C. A. STUART
}

(Communicated by Barbara L. Keyfitz)

\begin{abstract}
Under general hypotheses, we prove the existence of a nontrivial solution for the equation $L u=N(u)$, where $u$ belongs to a Hilbert space $H$, $L$ is an invertible continuous selfadjoint operator, and $N$ is superlinear. We are particularly interested in the case where $L$ is strongly indefinite and $N$ is not compact. We apply the result to the Choquard-Pekar equation
\end{abstract}

$$
-\Delta u(x)+p(x) u(x)=u(x) \int_{\mathbb{R}^{3}} \frac{u^{2}(y)}{|x-y|} d y, \quad u \in H^{1}\left(\mathbb{R}^{3}\right), u \neq 0,
$$

where $p \in L^{\infty}\left(\mathbb{R}^{3}\right)$ is a periodic function.

\section{INTRODUCTION}

Let $H$ be a real Hilbert space with scalar product $\langle\cdot, \cdot\rangle$ and norm $\|\cdot\|$. We consider in $H$ the equation

$$
L u=N(u), \quad u \neq 0,
$$

where $L: H \rightarrow H$ is an invertible continuous selfadjoint operator and $N$ is a superlinear operator whose properties are given in the next section. We suppose that $\sigma(L) \cap \mathbb{R}_{+} \neq \varnothing$. Denoting by $H_{+}$and $H_{-}$the eigenspaces of $L$ corresponding to $\sigma(L) \cap \mathbb{R}_{+}$and $\sigma(L) \cap \mathbb{R}_{-}$, respectively, there exists $\delta>0$ such that

$$
\forall u \in H_{+}:\langle L u, u\rangle \geq \delta\|u\|^{2}
$$

and

$$
\forall u \in H_{-}:\langle L u, u\rangle \leq-\delta\|u\|^{2} .
$$

We call $L$ strongly indefinite if the dimensions of $H_{+}$and $H_{-}$are infinite. The equation (1.1) has been considered in some recent papers [1, 5-8] (see also [3, $4,9-11$ ] for related problems). In this article we give a new existence theorem for (1.1). The proof given is simpler and the theorem can be applied to treat

Received by the editors September 16, 1991 and, in revised form, January 23, 1992.

1991 Mathematics Subject Classification. Primary 35J60, 49B40; Secondary 35A15, 35J10, $47 \mathrm{H} 15$.

Key words and phrases. Semilinear equation, Choquard-Pekar equation, strongly indefinite operator, lack of compactness. 
a larger class of nonlinearities. In particular, we can handle an equation of the form

$$
-\Delta u(x)+p(x) u(x)=u(x) \int_{\mathbb{R}^{3}} \frac{u^{2}(y)}{|x-y|} d y, \quad u \in H^{1}\left(\mathbb{R}^{3}\right), u \neq 0
$$

(the function $p \in L^{\infty}\left(\mathbb{R}^{3}\right)$ is periodic), which is referred to in the solid-state physics literature as a Choquard-Pekar equation. Note that the linear part of (1.4) is in general strongly indefinite. Due to the fact that the nonlinear part is not compact, the "linking theorems" of Benci-Rabinowitz and their generalizations, which played in [5-8] a crucial role in the discussion of (1.1), cannot be used. They require, indeed at a deep level, the compactness of the nonlinearity.

\section{THE GENERAL RESULT}

We suppose that there exists $\phi \in C^{2}(H, \mathbb{R})$ such that

(H1) $N=\nabla \phi$

(H2) $\lim _{\|u\| \rightarrow 0}\left(\phi(u) /\|u\|^{2}\right)=0$ and $\exists p>2, \forall u \in H:\langle N(u), u\rangle \geq p \phi(u)$.

(H3) $\phi$ is convex and $\phi(u)=0 \Rightarrow u=0$.

(H4) $\exists K, C>0$ such that $\forall u \in H:\|N(u)\| \leq K\langle N(u), u\rangle+C$.

(H5) If there exists a bounded sequence $\left\{u_{n}\right\} \subset H$ such that

$$
L u_{n}-N\left(u_{n}\right) \rightarrow 0 \text { and } \liminf _{n \rightarrow \infty}\left\langle N\left(u_{n}\right), u_{n}\right\rangle>0,
$$

then there exists $u \neq 0$ with $L u=N(u)$.

It follows from $(\mathrm{H} 2)$ that $\phi(0)=0, N(0)=0$, and the function

$$
\phi(t u) / t^{p}, \quad t>0,
$$

is increasing in $t$ for all $u \in H$. Together with (H3), this implies that

$$
\forall u \neq 0: \phi(u)>0 \text {. }
$$

Finally, if $N$ is compact then (H5) holds. Indeed, if $\left\{u_{n}\right\}$ satisfies the conditions of (H5), passing to a subsequence, we can suppose that $N\left(u_{n}\right) \rightarrow w$. Therefore $L u_{n} \rightarrow w$ and $u_{n} \rightarrow L^{-1} w:=u$. Clearly $L u=N(u)$ and $u \neq 0$.

Theorem 2.1. Under the hypotheses (H1) to (H5), there exists $u \in H$ such that $L u=N(u)$ and $u \neq 0$.

The idea of the proof is to find a nontrivial critical point of the function

$$
J(u)=\frac{1}{2}\langle L u, u\rangle-\phi(u) .
$$

The first step is a kind of Lyapunov-Schmidt reduction. Let $P$ denote the orthogonal projection onto $H_{+}$.

Lemma 2.1. There exists $g \in C^{1}\left(H_{+}, H_{-}\right)$such that

$$
\forall w \in H_{-}: w \neq g(v) \Rightarrow J(v+w)<J(v+g(v)) .
$$

Moreover,

$$
\operatorname{Lg}(v)=(I-P) N(v+g(v)) .
$$

Proof. For a given $v \in H_{+}$, the function $f_{v}$ defined on $H_{-}$by

$$
f_{v}(w)=\frac{1}{2}\langle L v, v\rangle+\frac{1}{2}\langle L w, w\rangle-\phi(v+w)
$$


is strictly concave and $\lim _{\|w\| \rightarrow \infty} f(w)=-\infty$. Hence there exists $g(v) \in H_{-}$ verifying (2.1). Since $g(v)$ is a critical point of $f_{v}$, it satisfies (2.2). Finally, the implicit function theorem shows that $g \in C^{1}\left(H_{+}, H_{-}\right)$. Indeed, $g(v)$ is the unique $w$ satisfying $L w-(I-P) N(v+w)=0$. The derivative of the left member with respect to $w$ is the operator defined on $H_{-}$by $d w \rightarrow$ $L d w-(I-P) N^{\prime}(v+w) d w$. This operator is selfadjoint; indeed,

$$
\begin{aligned}
\langle L d w & \left.-(I-P) N^{\prime}(v+w) d w, d z\right\rangle \\
& =\langle L d w, d z\rangle-\left\langle N^{\prime}(v+w) d w, d z\right\rangle \\
& =\langle L d z, w z\rangle-\left\langle N^{\prime}(v+w) d z, d w\right\rangle \\
& =\left\langle L d z-(I-P) N^{\prime}(v+w) d z, d w\right\rangle
\end{aligned}
$$

for all $d w, d z \in H_{-}$. Since

$$
\begin{aligned}
& \left\langle L d w-(I-P) N^{\prime}(v+w) d w, d w\right\rangle \\
& \quad=\left\langle L d w-N^{\prime}(v+w) d w, d w\right\rangle \\
& \quad \leq\langle L d w, d w\rangle \quad \text { by the convexity of } \phi \\
& \quad \leq-\delta\|d w\|^{2} \quad \text { by }(1.3)
\end{aligned}
$$

for all $d w \in H_{-}$, we can conclude that its inverse exists and is bounded.

Let us introduce the function $F$ defined on $H_{+}$by $F(v)=J(v+g(v))$. The next lemma allows us to restrict our attention to the critical points of $F$.

Lemma 2.2. $\forall v \in H_{+}:\|\nabla F(v)\|=\|\nabla J(v+g(v))\|$.

Proof. The relation (2.2) is equivalent to $(I-P) \nabla J(v+g(v))=0$. Hence we have for $d v \in H_{+}$:

$$
\begin{aligned}
\langle\nabla F(v), d v\rangle & =\langle\nabla J(v+g(v)), d v\rangle+\left\langle\nabla J(v+g(v)), g^{\prime}(v) d v\right\rangle \\
& =\langle\nabla J(v+g(v)), d v\rangle
\end{aligned}
$$

and so $\|\nabla F(v)\| \leq\|\nabla J(v+g(v))\|$. Conversely, for $d u \in H$,

$$
\begin{aligned}
\langle\nabla J & (v+g(v)), d u\rangle \\
& =\langle\nabla J(v+g(v)), P d u\rangle+\langle\nabla J(v+g(v)),(I-P) d u\rangle \\
& =\langle\nabla F(v), P d u\rangle
\end{aligned}
$$

and so $\|\nabla F(v)\| \geq\|\nabla J(v+g(v))\|$.

Proof of the theorem. The main ingredient in the proof is a version of the wellknown mountain pass lemma in which the Palais-Smale condition is not assumed [2, p. 943]. In order to use this result on $F$ we shall prove that

(1) $F(0)=0$;

(2) there exist $r, \alpha>0$ such that

$$
\forall v \in H_{+}:\|v\| \leq r \Rightarrow F(v) \geq 0 \text { and }\|v\|=r \Rightarrow F(v) \geq \alpha ;
$$

(3) there exists $v \in H_{+}$such that $F(v)<0$.

The first point is a direct consequence of $g(0)=0$. The first part of $(\mathrm{H} 2)$ and (1.2) imply the existence of $r, \alpha>0$ such that for all $v \in H_{+}$:

$$
\|v\| \leq r \Rightarrow \frac{1}{2}\langle L v, v\rangle-\phi(v) \geq 0
$$

and

$$
\|v\|=r \Rightarrow \frac{1}{2}\langle L v, v\rangle-\phi(v) \geq \alpha .
$$


Now (2.1) implies $F(v) \geq J(v)$, which proves the second point. Fix $v \in H_{+}$ with $\|v\|=1$. There exists $t>0$ such that $F(t v)<0$ (and so the third point holds). Indeed, consider a sequence $\left\{t_{n}\right\}$ such that $t_{n} \rightarrow \infty$. Setting $u_{n}=t_{n} v+g\left(t_{n} v\right)$ and $w_{n}=u_{n} /\left\|u_{n}\right\|$, we have $\left\|w_{n}\right\|=1$ and so, passing to a subsequence, $w_{n} \rightarrow w=w_{+}+w_{-}$, where $w_{ \pm} \in H_{ \pm}$. The subsequence can be chosen so that one of the following cases occurs: $\left\|g\left(t_{n} v\right)\right\| / t_{n} \rightarrow+\infty$ or $\left\|g\left(t_{n} v\right)\right\| / t_{n} \rightarrow \lambda \geq 0$. In the first case, for $n$ sufficiently large, we get

$$
\begin{aligned}
2 F\left(t_{n} v\right) & =2 J\left(t_{n} v+g\left(t_{n} v\right)\right) \\
& \leq t_{n}^{2}\langle L v, v\rangle-\delta\left\|g\left(t_{n} v\right)\right\|^{2} \quad \text { by }(1.3) \\
& <0
\end{aligned}
$$

In the second case, $t_{n} /\left\|u_{n}\right\| \rightarrow \mu=\left(1+\lambda^{2}\right)^{-1 / 2}>0$ and so $w=\mu v+w_{-} \neq 0$. Hence

$$
0<\phi(w) \leq \liminf _{n \rightarrow \infty} \phi\left(w_{n}\right)
$$

and there exists $n_{0}$ such that $\phi\left(w_{n}\right)>\frac{1}{2} \phi(w)$ for all $n \geq n_{0}$. Also

$$
\begin{aligned}
\phi\left(u_{n}\right) & =\phi\left(\left\|u_{n}\right\| w_{n}\right) \geq\left\|u_{n}\right\|^{p} \phi\left(w_{n}\right) \quad \text { by }(\mathrm{H} 2) \\
& \geq \frac{1}{2} t_{n}^{p} \phi(w)
\end{aligned}
$$

for $n \geq n_{0}$ since we may assume $\left\|u_{n}\right\| \geq t_{n} \geq 1$. Thus for $n \geq n_{0}$,

$$
2 F\left(t_{n} v\right)=2 J\left(u_{n}\right) \leq t_{n}^{2}\langle L v, v\rangle-t_{n}^{p} \phi(w)
$$

and so $F\left(t_{n} v\right)<0$ for $n$ sufficiently large.

Applying the mountain pass lemma, we see that there exists a sequence $\left\{v_{n}\right\} \subset H_{+}$such that $\lim _{n \rightarrow \infty} F\left(v_{n}\right)=c>0$ and $\nabla F\left(v_{n}\right) \rightarrow 0$. Setting $u_{n}=v_{n}+g\left(v_{n}\right)$, we have $\lim _{n \rightarrow \infty} J\left(u_{n}\right)=c>0$ and $\nabla J\left(u_{n}\right) \rightarrow 0$ by definition of $F$ and Lemma 2.2. Taking a subsequence, we can assume that $\left|J\left(u_{n}\right)-c\right| \leq 1 / n$ and $\left\|\nabla J\left(u_{n}\right)\right\| \leq 1 / n$. Now

$$
\begin{aligned}
\left\langle N\left(u_{n}\right), u_{n}\right\rangle & =\left\langle L u_{n}, u_{n}\right\rangle-\left\langle\nabla J\left(u_{n}\right), u_{n}\right\rangle \\
& =2 J\left(u_{n}\right)+2 \phi\left(u_{n}\right)-\left\langle\nabla J\left(u_{n}\right), u_{n}\right\rangle \\
& \leq 2\left(c+\frac{1}{n}\right)+\frac{2}{p}\left\langle N\left(u_{n}\right), u_{n}\right\rangle+\frac{\left\|u_{n}\right\|}{n} ;
\end{aligned}
$$

therefore

$$
\left\langle N\left(u_{n}\right), u_{n}\right\rangle \leq \frac{p}{p-2}\left(2 c+\frac{2}{n}+\frac{\left\|u_{n}\right\|}{n}\right)
$$

and so

$$
\begin{aligned}
\delta\left\|u_{n}\right\| & \leq\left\|L u_{n}\right\| \leq \frac{1}{n}+\left\|N\left(u_{n}\right)\right\| \\
& \leq \frac{1}{n}+C+K\left\langle N\left(u_{n}\right), u_{n}\right\rangle \quad \text { by }(\mathrm{H} 4) \\
& \leq \frac{1}{n}+C+K \frac{p}{p-2}\left(2 c+\frac{2}{n}+\frac{\left\|u_{n}\right\|}{n}\right) .
\end{aligned}
$$

This implies that $\left\{u_{n}\right\}$ is a bounded sequence. Finally

$$
\begin{aligned}
\left\langle N\left(u_{n}\right), u_{n}\right\rangle & =2 J\left(u_{n}\right)+2 \phi\left(u_{n}\right)-\left\langle\nabla J\left(u_{n}\right), u_{n}\right\rangle \\
& \geq 2\left(c-\frac{1}{n}\right)-\frac{\left\|u_{n}\right\|}{n}
\end{aligned}
$$


and therefore $\liminf _{n \rightarrow \infty}\left\langle N\left(u_{n}\right), u_{n}\right\rangle \geq 2 c>0$. The conclusion now follows from (H5).

\section{EXAMPLE}

We shall now apply the general theory developed in $\S 2$ to prove the existence of a weak solution for equation (1.4). The Hilbert space $H$ considered here is the Sobolev space $H^{1}\left(\mathbb{R}^{3}\right)$. The operator

$$
S: D(S) \subset L^{2}\left(\mathbb{R}^{3}\right) \rightarrow L^{2}\left(\mathbb{R}^{3}\right),
$$

defined by

$$
D(S)=H^{2}\left(\mathbb{R}^{3}\right) \text { and } S u=-\Delta u+p u
$$

with $p \in L^{\infty}\left(\mathbb{R}^{3}\right)$ periodic, is selfadjoint. Its spectrum is purely continuous, bounded from below and $\sigma(S) \cap \mathbb{R}_{+} \neq \varnothing$. We suppose that $p$ is such that $0 \notin \sigma(S)$. We associate with $S$ the operator $L$ defined on $H$ by

$$
\langle L u, v\rangle=\int_{\mathbb{R}^{3}}\{\nabla u \nabla v+p u v\} d x, \quad \forall u, v \in H .
$$

$L$ is selfadjoint, continuous, invertible and $\sigma(L) \cap \mathbb{R}_{+} \neq \varnothing$ (the relation between $S$ and $L$ is discussed in [3]).

In order to study the nonlinear part, we need the following remark. For $u \in L^{4 / 3}\left(\mathbb{R}^{3}\right) \cap L^{2}\left(\mathbb{R}^{3}\right), u \geq 0$, set

$$
f(x)=\int_{\mathbb{R}^{3}} \frac{u(y)}{|x-y|} d y .
$$

We have

$$
\begin{aligned}
f(x)= & \int_{|x-y| \leq 1} \frac{u(y)}{|x-y|} d y+\int_{|x-y| \geq 1} \frac{u(y)}{|x-y|} d y \\
\leq & \|u\|_{L^{2}\left(\mathbb{R}^{3}\right)}\left\{\int_{|x-y| \leq 1} \frac{1}{|x-y|^{2}} d y\right\}^{1 / 2} \\
& +\|u\|_{L^{4 / 3}\left(\mathbb{R}^{3}\right)}\left\{\int_{|x-y| \geq 1} \frac{1}{|x-y|^{4}} d y\right\}^{1 / 4} \\
\leq & C \max \left\{\|u\|_{L^{2}\left(\mathbb{R}^{3}\right)},\|u\|_{L^{4 / 3}\left(\mathbb{R}^{3}\right)}\right\} .
\end{aligned}
$$

Hence for all $u, v \in H$, we obtain

$$
\begin{aligned}
\int_{\mathbb{R}^{3}} & \left\{u(x) \int_{\mathbb{R}^{3}} \frac{u^{2}(y)}{|x-y|} d y\right\} v(x) d x \\
& \leq\|u v\|_{L^{1}\left(\mathbb{R}^{3}\right)} C \max \left\{\|u\|_{L^{4}\left(\mathbb{R}^{3}\right)}^{2},\|u\|_{L^{8 / 3}\left(\mathbb{R}^{3}\right)}^{2}\right\} \\
& \leq C\|u\|^{3}\|v\|,
\end{aligned}
$$

and therefore there exists $N(u) \in H$ such that

$$
\langle N(u), v\rangle=\int_{\mathbb{R}^{3}}\left\{u(x) \int_{\mathbb{R}^{3}} \frac{u^{2}(y)}{|x-y|} d y\right\} v(x) d x .
$$


The problem (1.4) consists now in finding $u \in H, u \neq 0$, such that $L u=N(u)$. In this aim, we define for $u \in H$

$$
\phi(u)=\frac{1}{4} \int_{\mathbb{R}^{3}} \int_{\mathbb{R}^{3}} \frac{u^{2}(x) u^{2}(y)}{|x-y|} d x d y .
$$

It remains to check the hypotheses (H1) to (H5). Thanks to (3.1), we have

$$
\phi(u) \leq\|u\|_{L^{2}\left(\mathbb{R}^{3}\right)}^{2} C \max \left\{\|u\|_{L^{4}\left(\mathbb{R}^{3}\right)}^{2},\|u\|_{L^{8 / 3}\left(\mathbb{R}^{3}\right)}^{2}\right\} \leq C\|u\|^{4}<\infty .
$$

Moreover, $\phi \in C^{2}(H, \mathbb{R}), \nabla \phi=N$, and

$$
\left\langle N^{\prime}(u) v, z\right\rangle=\int_{\mathbb{R}^{3}} \int_{\mathbb{R}^{3}} \frac{u^{2}(y) v(x) z(x)+2 u(y) v(y) u(x) z(x)}{|x-y|} d x d y .
$$

The hypothesis $(\mathrm{H} 2)$ is also easy to check.

Lemma 3.1. We have for all $u, v \in H$

$$
\int_{\mathbb{R}^{3}} \int_{\mathbb{R}^{3}} \frac{u(x) v(x) u(y) v(y)}{|x-y|} d x d y \geq 0
$$

and

$$
\begin{aligned}
& \left\{\int_{\mathbb{R}^{3}} \int_{\mathbb{R}^{3}} \frac{u^{2}(x) v^{2}(y)}{|x-y|} d x d y\right\}^{2} \\
& \quad \leq \int_{\mathbb{R}^{3}} \int_{\mathbb{R}^{3}} \frac{u^{2}(x) u^{2}(y)}{|x-y|} d x d y \int_{\mathbb{R}^{3}} \int_{\mathbb{R}^{3}} \frac{v^{2}(x) v^{2}(y)}{|x-y|} d x d y .
\end{aligned}
$$

Proof. Consider the bilinear form $a$ on $C_{0}^{\infty}\left(\mathbb{R}^{3}\right)$ that is defined by

$$
a(z, w)=\int_{\mathbb{R}^{3}} \int_{\mathbb{R}^{3}} \frac{z(x) w(y)}{|x-y|} d x d y .
$$

Clearly $a$ is symmetric. For $w \in C_{0}^{\infty}\left(\mathbb{R}^{3}\right)$ let

$$
g(x)=\int_{\mathbb{R}^{3}} \frac{w(y)}{|x-y|} d y
$$

be the corresponding Newtonian potential. Then $g \in C^{2}\left(\mathbb{R}^{3}\right)$ and

$$
-\Delta g(x)=4 \pi w(x) \quad \forall x \in \mathbb{R}^{3}
$$

and there exists $A>0$ such that

$$
|g(x)| \leq A|x|^{-1} \quad \text { and } \quad|\nabla g(x)| \leq A|x|^{-2} \quad \forall|x| \geq 1 .
$$

Hence,

$$
\begin{aligned}
a(w, w) & =\int_{\mathbb{R}^{3}} w(x) g(x) d x \\
& =-\frac{1}{4 \pi} \int_{\mathbb{R}^{3}} g(x) \Delta g(x) d x=\frac{1}{4 \pi} \int_{\mathbb{R}^{3}}|\nabla g(x)|^{2} d x \geq 0 .
\end{aligned}
$$

Also since $a$ is positive definite and symmetric on $C_{0}^{\infty}\left(\mathbb{R}^{3}\right)$, it follows that

$$
|a(w, z)| \leq a(w, w)^{1 / 2} a(z, z)^{1 / 2} .
$$

It is easy to conclude, using the density of $C_{0}^{\infty}\left(\mathbb{R}^{3}\right)$ in $H^{1}\left(\mathbb{R}^{3}\right)$ and (3.1). 
We deduce from Lemma 3.1 that $\left\langle N^{\prime}(u) v, v\right\rangle \geq 0$ for all $u, v \in H$. Consequently $\phi$ is convex. Moreover $\phi(0)=0 \Rightarrow u=0$ and (H3) is verified. For $u, v \in H$, we have

$$
\begin{aligned}
|\langle N(u), v\rangle| & =\left|\int_{\mathbb{R}^{3}} \int_{\mathbb{R}^{3}} \frac{u^{2}(y) u(x) v(x)}{|x-y|} d x d y\right| \\
& \leq\left\{\int_{\mathbb{R}^{3}} \int_{\mathbb{R}^{3}} \frac{u^{2}(x) u^{2}(y)}{|x-y|} d x d y\right\}^{1 / 2}\left\{\int_{\mathbb{R}^{3}} \int_{\mathbb{R}^{3}} \frac{v^{2}(x) u^{2}(y)}{|x-y|} d x d y\right\}^{1 / 2} \\
& \leq\langle N(u), u\rangle^{1 / 2}\langle N(u), u\rangle^{1 / 4}\langle N(v), v\rangle^{1 / 4} \text { by Lemma 3.1 } \\
& =\langle N(u), u\rangle^{3 / 4}\langle N(v), v\rangle^{1 / 4} \\
& \leq C\langle N(u), u\rangle^{3 / 4}|| v \| \text { by }(3.3) .
\end{aligned}
$$

Hence

$$
\|N(u)\| \leq C\langle N(u), u\rangle^{3 / 4} \leq C\langle N(u), u\rangle+C
$$

and (H4) is proved. Finally, let us consider a bounded sequence $\left\{u_{n}\right\} \subset H$ such that $L u_{n}-N\left(u_{n}\right) \rightarrow 0$ and $\liminf _{n \rightarrow \infty}\left\langle N\left(u_{n}\right), u_{n}\right\rangle>0$. If

$$
\lim _{n \rightarrow \infty} \sup _{y \in \mathbb{R}^{3}} \int_{y+B_{R}} u_{n}^{2} d x=0, \quad \forall R>0
$$

( $B_{R}$ denotes the open ball of radius $R$ centered at the origin), then by a result by Lions [13, Lemma I.1] $u_{n} \rightarrow 0$ in $L^{q}\left(\mathbb{R}^{3}\right)$ for all $q \in$ ]2, 6[ and, therefore, $\left\langle N\left(u_{n}\right), u_{n}\right\rangle \rightarrow 0$ by (3.2), which is a contradiction; otherwise, passing to a subsequence, we get

$$
\exists R<\infty, \quad\left\{y_{n}\right\} \subset \mathbb{R}^{3} \text { s.t. } \liminf _{n \rightarrow \infty} \int_{y_{n}+B_{R}} u_{n}^{2} d x>0 .
$$

Using the periodicity of $p$ and translating each $u_{n}$, we can find $R<\infty$ and a sequence $\left\{\tilde{u}_{n}\right\}$ such that

$$
L \tilde{u}_{n}-N\left(\tilde{u}_{n}\right) \rightarrow 0 \text { and } \liminf _{n \rightarrow \infty} \int_{B_{R}} \tilde{u}_{n}^{2} d x>0 .
$$

Passing to a subsequence, we have $\tilde{u}_{n} \rightarrow u$, and the compact inclusion $H^{1}\left(B_{R}\right) \subset L^{2}\left(B_{R}\right)$ shows that $u \neq 0$. Since $N\left(\tilde{u}_{n}\right) \rightarrow N(u)$, taking the limit in $L \tilde{u}_{n}-N\left(\tilde{u}_{n}\right) \rightarrow 0$, we obtain $L u=N(u)$. To establish that $N\left(\tilde{u}_{n}\right) \rightarrow N(u)$, set

$$
f_{n}(x)=\int_{\mathbb{R}^{3}} \frac{\tilde{u}_{n}^{2}(y)}{|x-y|} d y
$$

and define $f$ similarly with $\tilde{u}_{n}$ replaced by $u$. For $v \in H$,

$$
\left|\left\langle N\left(\tilde{u}_{n}\right)-N(u), v\right\rangle\right| \leq\left|\int_{\mathbb{R}^{3}}\left(\tilde{u}_{n}-u\right) f v d x\right|+\left\|\tilde{u}_{n}\right\|\left\|\left(f_{n}-f\right) v\right\|_{L^{2}\left(\mathbb{R}^{3}\right)} .
$$

Since $f v \in L^{2}\left(\mathbb{R}^{3}\right)$, we need only prove that $\lim _{n \rightarrow \infty}\left\|\left(f_{n}-f\right) v\right\|_{L^{2}\left(\mathbb{R}^{3}\right)}=0$. By estimates like (3.1), there exists $K>0$ such that $\left|f_{n}(x)\right| \leq K \forall x \in \mathbb{R}^{3}$ and $\forall n \in \mathbb{N}$. Also for all $R>0$

$$
\begin{aligned}
\left|f_{n}(x)-f(x)\right| & \leq(4 \pi R)^{1 / 2}\left\|\tilde{u}_{n}^{2}-u^{2}\right\|_{L^{2}(B(x, R))}+\left(\frac{4 \pi}{R}\right)^{1 / 4}\left\|\tilde{u}_{n}^{2}-u^{2}\right\|_{L^{4 / 3}\left(\mathbb{R}^{3}\right)} \\
& \leq K\left\{R^{1 / 2}\left\|\tilde{u}_{n}-u\right\|_{L^{4}(B(x, R))}\left\|\tilde{u}_{n}+u\right\|+R^{-1 / 4}\left(\left\|\tilde{u}_{n}\right\|^{2}+\|u\|^{2}\right)\right\}
\end{aligned}
$$


$\forall x \in \mathbb{R}^{3}$ and $\forall n \in \mathbb{N}$. Since the inclusion $H^{1}(B(x, R)) \subset L^{4}(B(x, R))$ is compact, this shows that the subsequence $\tilde{u}_{n}$ can be chosen so that $f_{n}(x) \rightarrow$ $f(x), \forall x \in \mathbb{R}^{3}$. Then by dominated convergence we can conclude that

$$
\lim _{n \rightarrow \infty}\left\|\left(f_{n}-f\right) v\right\|_{L^{2}\left(\mathbb{R}^{3}\right)}=0 \text {. }
$$

\section{ACKNOWLEDGMENT}

The authors thank A. Ambrosetti for fruitful discussions.

\section{REFERENCES}

1. S. Alama and Yan Yan Li, Existence of solutions for semilinear elliptic equations with indefinite linear part, J. Differential Equations 96 (1992), 89-115.

2. H. Brezis and L. Nirenberg, Remarks on finding critical point, Comm. Pure Appl. Math. 44 (1991), 939-963.

3. B. Buffoni and L. Jeanjean, Minimax characterization of solutions for a semilinear elliptic equation with lack of compactness, Ann. Inst. H. Poincaré Anal. Non Linéaire (to appear).

4. __ Bifurcation from the spectrum towards regular value, preprint.

5. H. P. Heinz, Lacunary bifurcation for operator equations and nonlinear boundary value problems on $\mathbb{R}^{N}$, Proc. Roy. Soc. Edinburgh Sect. A 118 (1991), 237-270.

6. __ Existence and gap-bifurcation of multiple solutions to certain nonlinear eigenvalue problems finalinfo preprint.

7. H. P. Heinz, T. Kupper, and C. A. Stuart, Existence and bifurcation of solutions for nonlinear perturbations of the periodic Schrödinger equation, J. Differential Equations (to appear).

8. H. P. Heinz and C. A. Stuart, Solvability of nonlinear equation in spectral gaps of the linearization, Nonlinear Anal. T.M.A. (to appear).

9. T. Kupper and C. A. Stuart, Bifurcation into gaps in the essential spectrum, J. Reine Angew. Math. 409 (1990), 1-34.

10. __ Bifurcation into gaps in the essential spectrum, 2, Nonlinear Anal. T.M.A. (to appear).

11. Gap-bifurcation for nonlinear perturbations of Hill's equation, J. Reine Angew. Math. 410 (1990), 23-52.

12. P. L. Lions, The concentration-compactness principle in the calculus of variations, Part 1, Ann. Inst. H. Poincaré Anal. Non Linéaire 1 (1984), 109-145.

13. The concentration-compactness principle in the calculus of variations, Part 2, Ann. Inst. H. Poincaré Anal. Non Linéaire 1 (1984), 223-283.

Département de Mathématiques, Ecole Polytechnique Fédérale de Lausanne, 1015 LAUSANNE, SWITZERLAND

E-mail address: stuart@masg3.epfl.ch 